\title{
O PERIGO DO MARKETING NO INCENTIVO À AUTOMEDICAÇÃO DE ANALGÉSICOS
}

Raisa de Moura Alves ${ }^{1}$

Thiago Pereira de Abreu ${ }^{2}$

RESUMO: Os Medicamentos são responsáveis em contribuir na saúde e bem estar do paciente, quando não são bem administrados á probabilidade a riscos e ocorrencias de eventos adversos, causando problemas ainda maiores ou a próprio óbito do paciente. E quando esses medicamentos são potencialmente perigosos, poderá atenuar o número maior de ocorrencias que ocasionarão prolongamento a complicações, gerenciando outras demandas ao uso de medicamentos sem prescrição médica. No Brasil, uma parte significativa relacionada ao uso inadequado a medicações, geram intoxicações ou demandam para outros tipos de complicações. Além disso, medicações para seu consumo é obrigatório ser prescritos e quando não há, acabam gerando problemas para os hospitais na qual gastam de 15 a $20 \%$ de seus orçamentos para resolver as complicações causadas pelo mau uso dos mesmos. A promessa de alívio do sofrimento rápido é inerente das propagandas e marketing de industrias farmacêuticas. As exigências para o uso racional de medicamentos são complexas, e para que sejam executadas, devem contar com a participação de todos, incluindo pacientes, profissionais de saúde, legisladores, formuladores de políticas públicas, indústria, comércio e governo.

Palavra-chave: Automedicação. Marketing. Indústrias farmacêuticas.

ABSTRACT: Medications are responsible for contributing to the patient's health and well-being, when they are not well administered to the probability of risks and events of adverse events, causing even greater problems or the patient's own death. And when these drugs are potentially dangerous, it may atheist the greater number of events that will prolong complications, managing other demands for the use of drugs without a prescription. In Brazil, a significant part related to inadequate use of medications, generate intoxications or demand for other types of complications. In addition, medications for their consumption are mandatory to be prescribed and

\footnotetext{
IFormação: Graduanda do curso de Farmácia. Universidade Iguaçu (UNIG)E-mail: e mouraraisa@gmail.com.

${ }^{2}$ Farmacêutico com doutorado em fisiologia. Instituição: UFRJ.
} 
when there are no, they end up generating problems for hospitals in which they spend 15 to $20 \%$ of their budgets to solve the complications caused by their misuse. The promise of relief from rapid suffering is inherent in the advertisements and marketing of pharmaceutical industries. The requirements for the rational use of medicines are complex, and for them to be executed, they must count on the participation of all, including patients, health professionals, legislators, policymakers, industry, commerce and government.

Keyword: Self-medication. Marketing. Pharmaceutical industries.

\section{INTRODUÇÃO}

Com as mudanças tecnológicas no século passado, levaram ao desenvolvimento das indústrias, principalmente de cunho laboratorial e farmacêutico, promovendo a síntese de novos compostos para diversas utilidades, incluindo os fármacos na qual o próprio consumidor adquirisse seus produtos sem a utilização e a obrigatoriedade de prescrição médica (MARGONATO et al., 2008).

A partir de 1920, as indústrias farmacêuticas desenvolveram fármacos com objetivo de contribuir com a saúde do indivíduo, através da eficácia existente quimicamente em seus produtos, na intervenção de doenças e com isso o avanço tecnológico contribuiu com o surgimento de antibióticos, vacinas e antihipertensivos. Embora que, a incorporação progressiva de novos fármacos trouxe à população outros problemas, como as enfermidades associadas à farmacoterapia por vezes agregada ao uso irracional dos medicamentos (TOURINHO,2008).

A Política Nacional de Medicamentos (Brasil, 1998) considera que o uso racional de medicamentos, compreende a prescrição certa ao tipo de gravidade relacionada a doença, e o consumo nas doses indicadas, nos intervalos definidos e no período de tempo indicado de medicamentos eficazes, seguros e de qualidade (DELFINO,2012).

A automedicação de cunho irracional pelo indivíduo, compreende o uso de medicamentos sem prescrição médica ou de um profissional qualificado em saúde. Esse conceito se difere da automedicação responsável, na qual o uso de medicamento não prescrito, porém, sob a orientação e acompanhamento do farmacêutico que irá favorecer uma conduta racional para o consumo desses fármacos (SALOMÃO, 200I). 
Infelizmente esse conceito é relacionado principalmente ao uso de fármacos sem prescrição médica, e um dos maiores responsáveis na colaboração de problemas oriundos a uso de medimentos, são direcionadas as empresas de produção de fármacos, na qual investem milhões em markenting na qual garante ao seu consumidor, produtos de qualidade, efeito rápido e solução para eliminação de dores com eficiencia e capacidade, nesse caso destaca-se os analgésicos (PAULA et al, 2021).

Os analgésicos são fármacos que tem a capacidade de reduzir ou aliviar a dor, dependendo de cada caso, os analgésicos se classificam como opiáceos e os não opiáceos. Os opiáceos são responsáveis em controlar a dor do paciente, até por que esse tipo de fármaco tem em sua composição quimica a morfina, encontra-se de forma natural ou sintetizado, nesse caso há obrigatoriedade da prescrição médica para o uso desse fármaco, até por que há risco de dependencia dessa medicação, e seus efeitos colaterias abrangentes consta a sonolência, náuseas, estado de euforia e mudança de comportamento no individuo, produzindo ansiedade e ao mesmo tempo depressão. A forma inadequada de seu uso poderá causar no indíviduo uma depressão respiratória grave e aguda, contribuindo até com a morte (SANTOS,2012).

Os não opiáceos são os fármacos de uso sem prescrição médica e que a industria farmaceutica prioriza como uma dos maiores fatores para ganho de consumidores em seu uso, pois viraliza em algo fácil, com rápido alivio e promovendo solução com eficacia, dando garantias quanto ao seu produto. Mas não sinaliza ao certo os cuidados que realmente deve ter ao uso continuo desse tipo de analgésico, e a necessidade de alertar sobre o uso de medicamentos sem prescrição médica (DELFINO,2012).

O protocolo de segurança na prescrição, uso e administração de medicamentos é fundamental para esclarecer e tirar dúvidas sobre o consumo desse fármaco e deverá ser aplicado em todos os estabelecimentos e propagandas que prestam cuidados à saúde, sinalizando de fato os riscos inerentes ao consumo sem a prescrição médica e que o seu uso é simplesmente para tratamento e medidas paliativas (AQUINO,2008). 
A falta qualificada de profissionais capacitados por conta das iniciativas governamentais, além de uma política de saúde irregular e inconstante, contribui pela falta adequada de orientação sobre a utilização de fármacos e contribui com a manutenção de índices elevados de intoxicações, na qual a cada dia tem se tornado constante em nossa sociedade. As propagandas e marketing das indústrias farmacêuticas atraem usuários e prescritores, e favorecem a utilização indevida de medicamentos por uma parcela importante da população (MARGONATO et al, 2008).

Ultimamente o consumo de fármacos pelos indivíduos tem cada vez mais sido alvo de interesse aos laboratórios farmacêuticos, contribuindo com o crescimento acelerado das indústrias voltados para esse público-alvo (PAULA et al,2021).

O crescimento dos gastos farmacêuticos pode ser resultado de muitos fatores como a ampliação de cobertura, surgimento de novos fármacos, dinamismo epidemiológico, envelhecimento da população, expectativas dos pacientes e o uso inadequado de fármacos em diversas situações clínicas (OMS, 2002).

A Organização Mundial de Saúde diz que:

"Há uso racional de medicamentos quando pacientes recebem fármacos corretos para suas condições clínicas e laboratorial, em doses adequadas às suas necessidades individuais, por um período adequado e ao menor custo para si e para a comunidade." (OMS, 2002).

Para que esse crescimento se torne constante para as indústrias farmacêuticas, muitas tem optado pelo investimento de propagandas, marketing e redes sociais quanto a apresentação de seus produtos, dando garantias e soluções imediatas quanto ao seu consumo, mesmo sem a prescrição médica. Mas na verdade, não distorcem quanto ao uso de medicamentos sem a orientação adequada ao paciente, que pelo comodismo oferecido dispensam as consultas clínicas, contribuindo aos problemas relacionados com medicamentos, que além de interferir na evolução clínica dos pacientes, são "culpados" por um número alarmante de hospitalizações e mortes a cada ano, provocando um aumento considerável dos custos dos sistemas de saúde, quanto ao uso inadequado (ROMANO-LIEBER,2002).

Assim, é fundamental que o uso de fármacos seja prescrito corretamente, de uma linguagem clara e farmacêutica, doses e período de duração do tratamento; que 
esteja disponível de modo apropriado, a um preço considerável na qual o paciente possa ter condições financeiras de manter o seu tratamento, que os critérios de qualidade obrigatórios; que se dispense em condições adequadas, com a orientação médica ou de um Farmacêutico; que se cumpra a terapia já prescrita, da melhor maneira possível. Conceito semelhante também é proposto pela Política Nacional de Medicamentos (BRASIL, 200I).

Muitos médicos acabam associando aos laboratórios de cunho farmacêutico, e não têm acessibilidade sobre informações completas a respeito da segurança dos fármacos que são administradas aos seus pacientes. Outros desconhecem os possíveis efeitos nocivos do que prescrevem, ou são leigos em observar que determinados fármacos aos associados a outros, podem gerar combinações perigosas entre as substâncias. Por outro lado, alguns pacientes não declaram estar usando outro tipo de fármacos que muitas das vezes foi por indicação de amigos ou ter buscado nas redes sociais, principalmente ao Google que hoje é considerado uma das maiores fontes de informação rápida no mercado. Há também aqueles acompanhados por vários médicos sem a intercomunicação entre eles (NASCIMENTO, 2003).

Ação mais séria no controle da legislação, produção, venda e propaganda de medicamentos precisa ser colocada em prática para que a população não seja afetada por efeitos maléficos ou que dele se tornem vítimas (LUCCHESI et al., 2005). Isso, porque, além do efeito terapêutico, os medicamentos têm o potencial de produzir efeitos adversos, colaterais e tóxicos. $\mathrm{O}$ uso irracional de medicamentos envolve diversos fatores de posição cultural, social e governamental (PORTA,2006).

\section{OBJETIVOS}

\section{OBJETIVO GERAL}

Evidenciar que o uso inadequado de medicamentos sem prescrição médica, indiscriminado, abusivo, poderá causar diversos problemas a saúde. 


\section{OBJETIVOS ESPECÍFICOS}

- Desenvolver práticas seguras no uso de medicamentos em estabelecimentos de saúde.

- Identificar os danos causados por uso inadequado de medicamentos.

- Esclarecer que o uso irracional poderá favorecer uma situação de morbimortalidade.

\section{METODOLOGIA}

Este estudo foi desenvolvido em revisões bibliográficas, através de consulta em revistas, artigos científicos nacionais e internacionais indexados, dissertações, monografias, teses, bases de dados eletrônicas da U.S. Nattional Libray of Medicine and The National Institutes of Health (PubMed), Biblioteca Virtual em Saúde (EDENF,LILACS, MEDLINE), Scientific Electronic Library Online (SciELO) e Google Acadêmico.

\section{JUSTIFICATIVA}

Ultimamente o consumo de fármacos pelos indivíduos tem cada vez mais sido alvo de interesse aos laboratórios farmacêuticos, contribuindo com o crescimento acelerado das indústrias voltados para esse público-alvo.

As principais causas referentes ao uso inadequado de medicações, desenvolvem reações adversas aos medicamentos (RAM), não adesão ao tratamento, super dosagem ou subdosagem, falta da farmacoterapia necessária, inadequado seguimento de sinais e sintomas, e erros de medicação (CARVALHO, 2007).

O consumo inadequado, a automedicação e a indicação de remédios por pessoas não capacitadas tecnicamente são um grave problema de saúde pública no Brasil ultimamente, pois a falta de conhecimento e a ignorância em igualar sintomas parecidos com respectivas doenças, sem de fato reconhecer a sua procedência, tem um sério risco de morte no indivíduo, pois cada caso é um caso diferenciado, ou seja, nem sempre uma dor de cabeça está relacionado a um tipo de doença, podendo ser 
algo com complexidade ainda maior e necessita de exames laboratoriais específicos quanto a descoberta de fato do que se trata.

Esse estudo de pesquisa busca desenvolver uma visão crítica sobre as propagandas e marketing de indústrias farmacêuticas que apresentam seus produtos de uma forma ilusória quanto ao seu uso, sem de fato apresentar as demandas que se encontram por trás de cada rótulo.

\section{DESENVOLVIMENTO}

\section{I Contexto Histórico}

Em 1882 foi o ano que surgiu o primeiro anuncio de medicamentos em propaganda nos jornais, na qual era considerado o primeiro produto industrializado no Brasil é que precisava ser divulgado como uma das melhores descobertas do cunho farmacêutico, a pomada Boro-borácica, responsável para tratamentos na pele e mucosa (LUCHESSI et al,2005). Uma propaganda também que ficou muito tempo registrada, foi o anuncio em revistas do Xarope São João em I9oo, originaria do Rio de Janeiro, na qual a sua imagem estava vinculada a de um homem amordaçado, que representava a tosse seca, a bronquite que estava o sufocando, garantindo ao consumidor alivio rápido e se referia ao xarope como salvador dessa agonia (PAULA et $\mathrm{al}, 2020)$.

O Código de Defesa do Consumidor e o Conselho Nacional de Auto Regulação Publicitária (CONAR), garante ao consumidor que não seja mais enganado com propagandas que oferecem algum tipo de multifuncionalidade além do que realmente o produto fornece, como o próprio anuncio do Xarope São João que era próprio para o alivio da tosse, mas que também tinha funcionalidade em relação a problemas de reumatismos e quedas excessivas de cabelo, e que na verdade era apenas um produto feito com algumas ervas medicinais que proporcionava desempenho contra a tosse (LUCHESSI et al,2005).

O marketing sempre foi a alma de qualquer negócio, e principalmente nos tempos antigos onde a informação era realmente prestada pela questão do senso comum entre as pessoas, alguns depoimentos feitos por supostos ex pacientes que 
teriam conseguido a cura através do uso do medicamento em anúncio, também era outra forma de propaganda de medicamentos muito utilizada no fim do século XIX e início do século XX (PAULA et al,2020).

As propagandas publicitárias antes da década de 30, era totalmente informativa e escrita com uma linguagem formal e conotativa, destacando sempre a ilustração do produto com certo exagero para transmitir as propriedades de cura do medicamento. Destacando que nesse período, os redatores publicitários não tinham uma formação especializada na linguagem publicitária ou comercial, na realidade eram escritores literários ou poetas brasileiros. Como por exemplo destaca-se: Casemiro de Abreu, Lopes Trovão, Emílio de Meneses, Basílio Viana, Olavo Bilac e Orígenes Lessa, que eram voltados para a literatura, poesia e sem nenhum conceito relacionado diretamente a produção de propagandas, ainda mais relacionado a produtos farmacêuticos, mas a literatura tinha um papel fundamente na época de alcançar um público culto e intelecto (MACEDO,2016).

A divulgação de propaganda e marketing ganhou força a partir de 1930, com o surgimento da Rádio Nacional, onde as indústrias farmacêuticas ganharam forças ao contribuir com capital as rádios para divulgarem seus medicamentos, e onde os anúncios passaram a ter uma característica mais moderna, e como a utilização do tempo no rádio era limitada, as propagandas tinham como objetivo serem rápidas e objetivas ou usavam vozes de cantores para divulgarem os slogans do produto, como por exemplo o medicamento Melhoral que até os dias atuais as pessoas ainda comentam "Melhoral é melhor e não faz mal" (LUCHESSI et al,2005).

Emig6o, após um período de crescimento e desenvolvimento para a propaganda de medicamentos, houve uma redução no quantitativo de anúncios, devido ao controle de preços que passou a ser exercido sob todos os fármacos (MACEDO,2016). A partir da década de 1970, os anúncios e propagandas se fortaleceram novamente, mas de uma forma branda, reduzida e com cuidados. Esses anúncios eram veiculados principalmente na televisão, que chegou ao Brasil na década de 1950, e com o passar do tempo evoluíram e conquistaram a confiança e os investimentos das indústrias farmacêuticas em propaganda (BUENO,2017). 
Uma grande conquista na hegemonia da propaganda de fármacos foi a regulamentação, através da Lei da Vigilância Sanitária de no 6.360 de setembro de 1976, de vários aspectos acerca dos medicamentos, inclusive a propaganda, se transformando em um marco significativo para as indústrias farmacêuticas (LUCHESSI et al,2005).

Onde os medicamentos passaram a ter uma divisão distinta, ou seja, todo os tipos de fármacos que eram vendidos sob prescrição, não puderam mais ser anunciados em propagandas, rádios ou qualquer meio de comunicação que divulgasse um produto que só poderia ser consumido com a autorização médica, e nesse contexto surgem os profissionais de propaganda farmacêutica.

Esses profissionais promoveriam a propaganda dos medicamentos com venda sob prescrição. Uma vez que as propagandas desses medicamentos deveriam se restringir diretamente a profissionais prescritores ou a anúncios em publicações específicas dos laboratórios e da indústria farmacêutica (MACEDO,2016).

\subsection{A influência do marketing sobre a vida do consumidor}

A propaganda se fortaleceu ainda mais com o desenvolvimento tecnológico na sociedade, onde as redes sociais se tornaram uma fonte crescente de buscas e formas de se interagir, principalmente relacionado as propagandas de fármacos, que desempenharam um papel forte em divulgar os seus produtos, dando qualidade, rapidez e eficiência quanto a qualquer tipo de doenças. Infelizmente o interesse econômico acima do interesse a saúde é um dos grandes problemas no Brasil. Apesar de se ter outras estratégias a não ser somente o interesse pela venda do medicamento, a realidade que é encontrada é o interesse pelo consumo da população para o lucro das empresas (LUCHESSI et al,2005).

Estamos em uma época que a cada dia as pessoas se tornam reféns do que consomem, ou estão propícios a vulnerabilidade do estresse diário na qual provocam em sua rotina a produção de insônia, depressão devido ao ritmo acelerado de vida. Neste caso, a uma maior busca da população pelos medicamentos "milagrosos" que muitas das vezes as publicidades e propagandas idealizam e vendem este sonho, mas que não revelam de fato o que além dos milagres proposto podem induzir na vida do 
indivíduo, como por exemplo a sua dependência ao produto, de acordo com as composições químicas que são atribuídas a esse tipo de fármaco (AKERMAN,2017).

A propaganda tenta vender um universo idealizado, onde o medicamento pode levar a busca do bem estar e com qualidade de vida de uma forma eficaz e rápida, aliviando seus problemas com qualidade através do consumo de seus “milagrosos comprimidos" (figura I). O ser humano tem uma tendência de não querer sofrer e de tratar as dores sem mesmo buscar alternativas, aonde nesta fragilidade a propaganda reforça o consumo do medicamento sem necessidade e sem orientações necessárias para o indivíduo (BERMUDEZ,2016).

Figura I: Os principais prescritores leigos e informais no Brasil
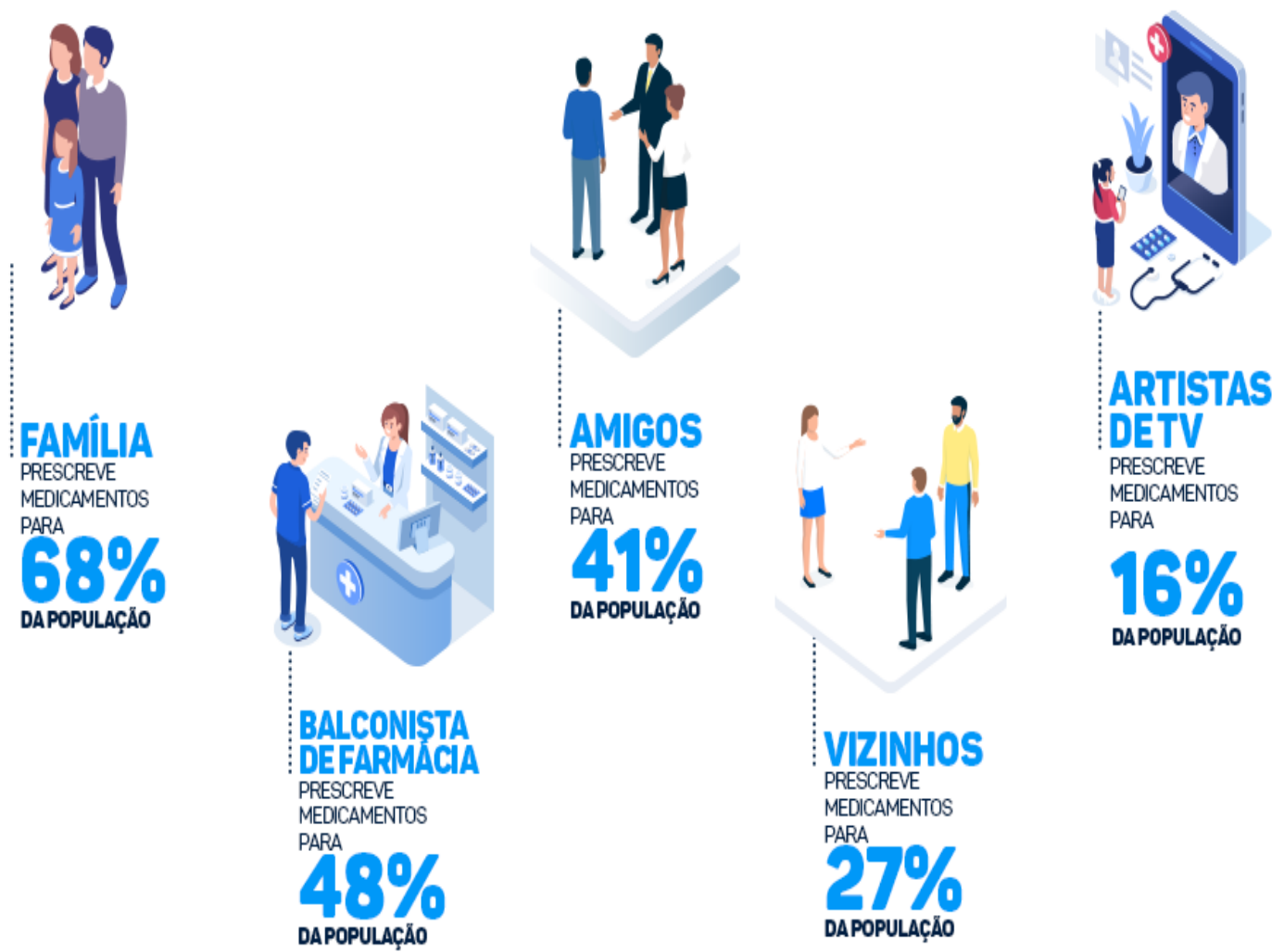

Fonte: ICQT 2018 
Todo medicamento livre de receitas médicas, não são livres de reações adversas e efeitos colaterais, independente do que está proposto a oferecer. Se for consumido de maneira equivocada ou indiscriminados sem orientação, pode trazer sérias complexidades a saúde ou até levando ao paciente ao óbito, pois existe algumas reações adversas que nem todo individuo está propenso a ter, ainda mais se tiver algum tipo de alergia, como o próprio zinco que em alguns pacientes produzem reações alérgicas.

Nenhum medicamento é zero de risco à saúde, este possui contra indicação que deve ser evidenciada e esclarecida ao consumidor, mas infelizmente a população busca a automedicação para sanar problemas que consideram coisas simples, como por exemplo o alívio da dor (figura 2) (BUENO,2017).

Figura 2: Dor de cabeça, febre e resfriado lideram entre os sintomas que levam as pessoas a tomar remédios por conta própria

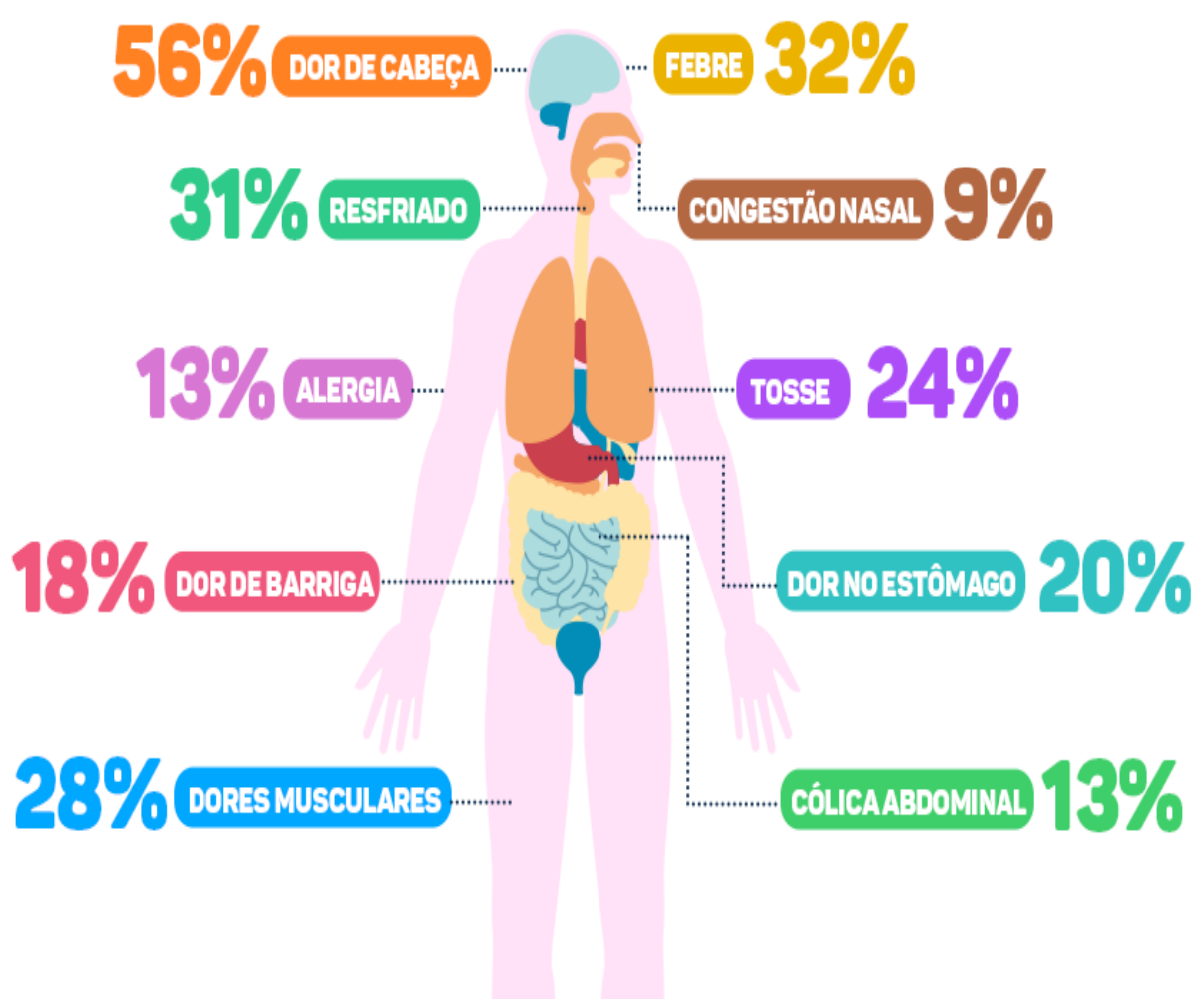

Fonte: ICQT 2018 
Uma das formas de propaganda e marketing que possui acesso rápido ao consumidor são os meios de comunicação com a capacidade de levar uma informação desejada a público. A grande maioria da população possui algum acesso a este meio, desde os meios de comunicação como rádio, tv, jornais e revistas ao acesso a redes sociais que tem buscado intensificar suas propagandas de forma mais visível e acessível a população, onde torna mais atrativas e lucrativas esta utilização por parte das indústrias farmacêuticas, pois atinge um público bem maior (MACEDO,2016).

Este é um dos grandes riscos pois a informação prestada nem sempre é confiável, principalmente quando uma variedade de fármacos garante qualidade de vida ao consumidor e que não há restrição ao seu uso, e que garante resultados. Mas esse tipo de informações acaba chegando de uma maneira rápida, atingindo uma grande parte da população em massa, e os números de danos também podem ser desastrosos. Por isso se dar a importância de uma fiscalização mais rigorosa quanto às informações de medicamentos que são levadas ao público, independente do que realmente ele seja, desde ao xarope ao analgésico (BUENO,2017).

Para Arrais et al,(2016) a influência pelo consumo onde as pessoas passam a consumir porque indicaram numa propaganda que tal medicamento é o correto, é levar a população ao uso indiscriminado de medicamentos sem qualquer orientação profissional de Medicina ou Farmácia. É colocar em risco a saúde pública para fins lucrativos e não para o bem da saúde.

\subsection{Os perigos da automedicação por analgésicos}

A automedicação já é algo que se tornou cultural na sociedade, onde um individuo por ser curado de uma enfermidade através de um fármaco, se acha capacitado em divulgar ao outros que possuem algo semelhante a consumir o mesmo. E na verdade é algo muito perigoso, pois cada organismo tem sua forma de receber um determinado fármaco e responder conforme a sua imunidade, e conforme não produza resultados positivos, poderá levar a pessoa a morte (SANTOS,2012).

O predomínio do uso de analgésicos (figura 3), é uma questão também de gênero, observa-se em estudos que as mulheres consomem muito mais que os homens, por isso as propagandas relacionadas a uso desse tipo de fármacos estão cada 
vez voltada as mulheres (AKERMAN,2017). Sinalizando que nenhum fármaco é totalmente inócuo, a questão de automedicar pode ser algo desfavorável à saúde individual e coletiva, pois não sabe ao certo do que se trata e ao invés de procurar pelo menos um farmacêutico e tentar esclarecer sobre o consumo do produto, preferem observar as propagandas e acreditar nelas, se tornando refém da sua insegurança (BUENO,2016).

Figura 3: Os analgésicos no ranking dos medicamentos mais consumidos pela população brasileira.

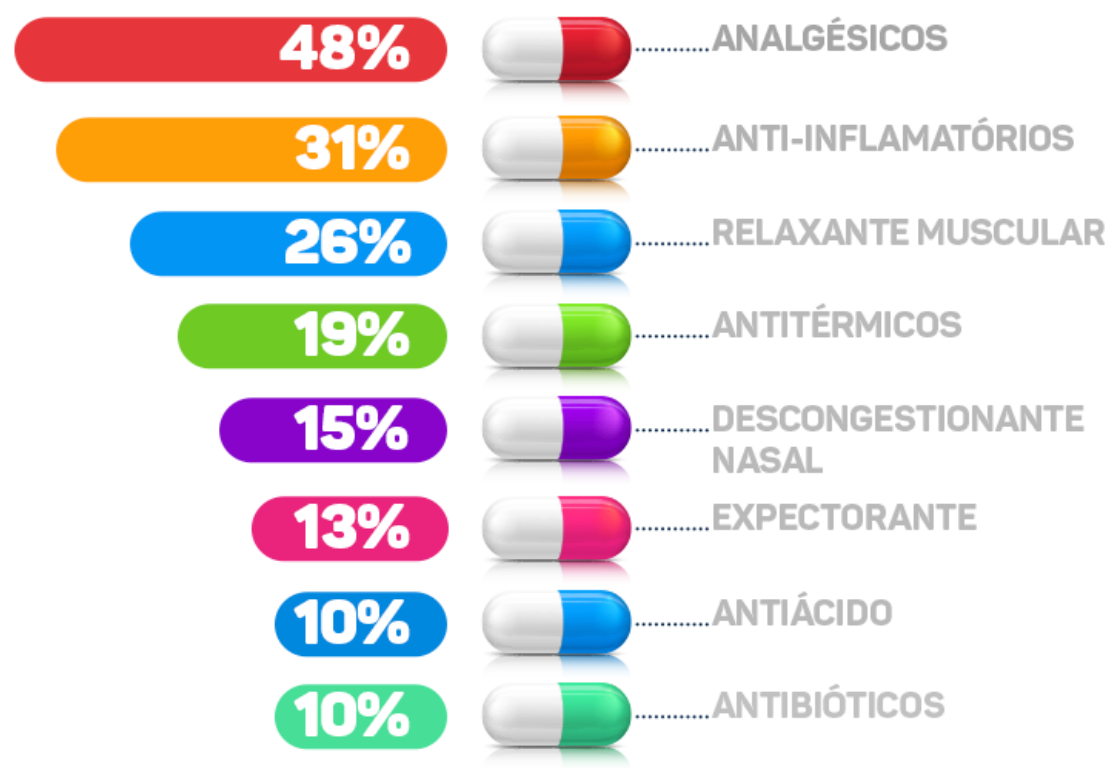

Fonte: ICQT 2018

A utilização indevida por uso contínuo de analgésicos, pode promover vários efeitos, como: reações de hipersensibilidade, estimulo para geração de anticorpos sem a merecida necessidade, dependência do medicamento sem precisão real, hemorragias digestivas, entre outros. E também a capacidade de desenvolver a intoxicação por uso excessivo desse produto (GERMANO et al,2012).

Um dos maiores problemas desenvolvidos por automedicação é a intoxicação por medicamentos, sendo esse responsável por grande parte das mortes no Brasil. Além disso, o alívio momentâneo dos sintomas pode mascarar a doença de base, 
podendo se agravar o estado de saúde do indivíduo e levando ao óbito. A automedicação se transformou em nossa sociedade em uma prática permanente, e para isso é necessário elucidar informações a sociedade sobre os fármacos de venda livre, sem estimular o consumo desenfreado ou o mito de cura milagrosa, que é o objetivo do marketing (GANDOLFI,2006).

Os analgésicos são um produto de venda livre, ou seja, são permitidos segundo as normas sanitárias, com a finalidade de aliviar sintomas e problemas menores de saúde, contando que sejam utilizados segundo as informações contidas no produto, mas na verdade são de fato ignoradas pelo consumidor, que muita das vezes os problemas persistem e ao invés de buscar ajuda ao profissional de saúde, preferem se automedicarem com outros tipos de fármacos, agravando ainda mais o problema (SANTOS,2012).

As propagandas de medicamentos encorajam a população a empregar a prática da automedicação ao acrescentar no fim das propagandas o dito "persistindo os sintomas um médico deverá ser consultado", excluindo desta maneira o produto anunciado de qualquer compromisso com a saúde. Apesar de no Brasil a ANVISA normatizar para o comércio e publicidade de medicamentos isento de prescrição, ainda faltam orientações necessárias para os que praticam a automedicação (GONÇALVES et al,2017).

\section{DISCUSSÃO}

O uso descontrolado de medicamentos isentos de prescrições é um problema de saúde pública prevalente em todo o mundo, e a propaganda é um dos meios de informações que as indústrias farmacêuticas desenvolveram para atrair consumidores, mas que se tornou um dos grandes violóes relacionado ao uso inadequado de medicamentos, principalmente os analgésicos.

Em sua pesquisa sobre automedicação, Akerman e Freitas (2017), consideraram os resultados dentro de uma faixa de magnitude considerada aceitável, embora que, conforme constataram em seu estudo, o uso de inadequado de medicamentos por automedicação, desenvolveram problemas ao tratar de doenças agudas autolimitadas, como problemas no estômago ou intestino, febre, dor, gripe, 
resfriado ou rinite alérgica, náusea e vômito, entre outros, pois ao perceberem que não produziam alivio as suas demandas, misturavam outros tipos de fármacos para tentar sanar seu problema.

Para Macedo et al. (2016), a automedicação é algo que desenvolveu na sociedade moderna como um costume e pode estar relacionada tanto a problemas quanto a soluções em relação aos cuidados com a saúde das pessoas, pois o seu atrativo está relacionado ao alívio rápido e prolongado, garantindo qualidade a saúde ao seu consumo, pois não provocam reações adversas, mas que ao persistir procurar um médico. Por isso as pessoas se acomodam e acreditam que esses tipos de fármacos são uma solução em momentos de emergências e ignoram o fato de ser algo mais complexos.

Não há como extinguir a automedicação da sociedade, em contra partida, existe recursos capazes de minimizar seus riscos e gravidades (BERMUDEZ E BARROS 2016). Para Macedo et al. (2016), de acordo com a Associação Brasileira de Medicamentos Isentos de Prescrição (ABIMIP), considera que a sociedade está acostumada culturalmente à prática da automedicação para eliminar ou melhorar sintomas comuns, como por exemplo, os sintomas gripais, dores musculares, dores de cabeça e de garganta, tosse, e até mesmo, prisão de ventre, assaduras, azias e má digestão, relacionado principalmente ao uso de analgésicos para aliviar esses tipos de sintomas (ARRAIS et al,2016).

Segundo Vieira e Zucchi (2013), o uso racional de medicamentos no Brasil, passou por muitas transformações relacionado a saúde populacional, através de investimentos financeiros e de infraestrutura para aumentar a oferta de medicamentos aos serviços de saúde, principalmente na área da atenção primária, com a Estratégia Saúde da Família.

Segundo Bermudez e Barros (2016), relacionado aos resultados do inquérito domiciliar abrangente da PNAUM (Pesquisa Nacional sobre Acesso, Utilização e Promoção do Uso Racional de Medicamentos no Brasil), onde coletaram informações em relação a vários aspectos do acesso e uso de medicamentos, perceberam que existe gastos exorbitantes com a saúde por conta de intoxicação, devido a prática da automedicação. 
Para Gonçalves et al. (2017), as intoxicações por medicamentos ocorrem por vários motivos, dentre os principais destacam-se a administração acidental, ao uso inadequado de medicações, as tentativas de suicídio e o abuso (principalmente entre adolescentes e adultos), além dos erros de administração.

Germano e Alonso (2017) citam alguns fatores que geralmente estão relacionados às intoxicações medicamentosas, sendo eles: a automedicação, o erro de prescrição, a exposição acidental, a utilização inadequada e, dentre esses fatores, os que se destacam são: as tentativas de suicídio, aborto e homicídio. O suicídio é considerado como a principal causa das intoxicações medicamentosas, justificada pelo uso em altas doses e pelo uso irracional de medicamentos, sendo mais prevalente entre mulheres jovens.(GANDOLFI; ANDRADE, 2006).

De acordo com Ribeiro et al. (2020), dados do Ministério da Saúde, revelam que nos anos de 20II - 2020, houve um aumento abusivos de casos por intoxicação notificados no Brasil pelo SINAN (Sistema de Informação de Agravos de Intoxicação). Sendo considerado a automedicação como um fator significativo nas tentativas de suicídio, o uso de medicamentos foi predominante nesse caso, na qual o individuo adquire o produto e como não existe prescrição controlada, consegue obter um quantitativo ainda maior para o seu consumo.

$\mathrm{Na}$ pesquisa de Arrais et al. (2016), sobre automedicação, os analgésicos e os relaxantes musculares foram os fármacos mais consumidos sem prescrição médica, sendo utilizado não apenas por crianças, mas um quantitativo de idosos que sofrem inúmeras complexidades de saúde, e para sanar seus problemas consomem medicamentos em excesso, até porque eles acreditam no que realmente são expostos pelas indústrias farmacêuticas em suas propagandas.

Ribeiro et al. (2020), considera em seus estudos que inúmeros casos relacionados a saúde desenvolveram complexidades devido ao uso de analgésicos, contribuindo para que a doença se tornasse ainda mais complexa, ou seja, pacientes buscaram ajuda de um profissional de saúde quando de fato a doença já apresentava em estado avançado, nesse caso destaca-se consumidores com diabetes, hipertensos, renais e até com câncer. 
Para Vieira et al, (2013), há necessidades urgente de desenvolver meios para contribuir com orientações aos profissionais de saúde e a população em geral, ou seja, projetos de encorajamento à busca de profissionais médicos e/ou farmacêuticos e expansão de políticas públicas a fim de adaptação de infraestrutura e supervisionamento adequado, da propagação em publicidade e da venda de medicamento isentos de prescrição, são medidas essenciais que ajudam a minimizar os males da automedicação.

\section{CONCLUSÃO}

A automedicação por analgésicos é algo que deve ser tratado como uma demanda séria, e que órgãos competentes devem buscar soluções e meios para esclarecer a população sobre o seu risco, na qual aparentemente são nocivos e apenas como um paliativo para aliviar a dor de forma rápida e por um determinado tempo de acordo com o marketing apresentado por cada indústria farmacêutica, aonde o capital é considerado importante para cada empresa.

O grande número de medicamentos desnecessários que é disponibilizado a população brasileira, coopera para que exista vários problemas relacionados ao uso de medicamentos sem orientações, por esta razão os casos de intoxicações se tornaram as maiores causas de agravos a saúde dos indivíduos. Principalmente ao uso descontrolado de fármacos é altamente comum, mas que gera complexidades na vida do consumidor independente do seu nível de escolaridade ou pela classe social. Outro fator é a privação de fundos orçamentários propostos ao Sistema Único de Saúde.

A falta de conhecimento pela eficácia de um determinado tipo de medicamento, deve ser acompanhada e esclarecida pela assistência farmacêutica, na qual direcionará condutas sobre as orientações necessárias para um atendimento eficaz e seguro. Para isso, o farmacêutico tem a capacidade de produzir fichas de acompanhamento farmacoterapêutico, guias explicativos e dedicar-se a educação continuada de seus clientes. A orientação é fundamental para qualquer atendimento, principalmente em algo relacionado a saúde, pois vários usuários não possuem conhecimento sobre os intervalos de administração dos medicamentos, o que pode interferir na biodisponibilidade do medicamento e, consequentemente, em sua 
efetividade farmacológica. $O$ indivíduo que detém conhecimento colabora para eficácia terapêutica e adota medidas profiláticas reduzindo os problemas associados ao uso de produtos farmacêuticos.

\section{REFERENCIAS}

AGÊNCIA NACIONAL DE VIGILÂNCIA SANITÁRIA - ANVISA. Farmacovigilância. Disponível em: http://portal.anvisa.gov.br. Acessado em: o7 agosto 2021.

ARAÚJO, ALA. Perfil da assistência farmacêutica na atenção primária do Sistema Único se Saúde,202I. Disponível em: http://scielo.com.br. Acesso: Io agosto 202I.

ARRAIS, Paulo Sergio Dourado. FERNANDES, Maria Eneida Porto. SILVA, Tatiane Dal Pizzoll da. RAMOS, Luiz Roberto. MENGUE, Sotero Serrate. LUIZA, Vera Lucia. TAVARES, Noemia Urruth Leão. FARIAS, Mareni Rocha. OLIVEIRA, Maria Auxiliadora. BERTOLDI, Andréa Damaso. Prevalência da automedicação no Brasil e fatores associados. Rev Saude Publica. 2016;50(supl 2):13s.

ARRAIS, P. S. D. et al. Perfil da automedicação no Brasil. Revista de Saúde Pública. São Paulo, v.3I, n.I, p.71-77,1997. Disponível em: https://www.scielo.br/scielo.php?pid=So0348910199700oIoooro\&script=sci_arttext Acesso em: 15 ago. 2021.

AQUINO, Daniela Silva de. Por que o uso racional de medicamentos deve ser uma prioridade?. Ciência \& Saúde Coletiva [online]. 2008, v. 13, n. suppl [Acessado 23 Agosto 2021] , pp. 733-736. Disponível em: <https://doi.org

AKERMAN, M.; FREITAS. O. Pesquisa Nacional sobre Acesso, Utilização e Promoção do Uso Racional de Medicamentos (PNAUM): avaliação dos serviços de atenção farmacêutica primária. Revista de Saúde Pública, v.5I, supl.2, 2017. 
Disponível em: http://www.revistas.usp.br/rsp/article/view/139702/135171 Acesso em: Io ago. 202I

BERMUdEZ, J. A. Z.; BARROS, M. B. A. Perfil do acesso e da utilização de medicamentos da população brasileira: contribuições e desafios da PNAUM Inquérito Domiciliar. Revista de Saúde Pública. v.50, supl 2, 2016. Disponível em: https://doi.org/10.1590/Si518-8787.20160500osupl2ap Acesso em: I5 ago. 2021.

BRASIL. MINISTÉRIO DA SAÚdE. SECRETARIA DE POLÍTICAS DE SAÚDE. Política Nacional de Medicamentos. Brasília: Ministério da Saúde, 2001 . 4op. Disponível

em: http://bvsms.saude.gov.br/bvs/publicacoes/politica_medicamentos.pdf Acesso em: I5 ago. 2021.

BRASIL. MINISTÉRIO DA SAÚDE. Consulta Pública no 95 , de i9 de novembro de 200r. Brasília: Agência Nacional de Vigilância Sanitária, 20orb. Disponível em: http://www4.anvisa.gov.br/base/visadoc/CP/CP\%5B2735-1-0\%5D.PDF Acesso em: Io set. 202I.

BRASIL. MINISTÉRIO DA SAÚdE. AGÊNCIA NACIONAL DE VIGILÂNCIA SANITÁRIA. Resolução $N^{\circ} .138$, de 29 de maio de 2003. Dispõe sobre o enquadramento de venda de medicamentos. Brasília: Anvisa, 2003. Disponível em: https://www.cff.org.br/userfiles/33\%20\%20BRASIL_\%20MINIST\%c3\%89RIO\%20DA\%20SA\%c3\%9aDE\%202003\%20RDC _I38_2003_ANVISA.pdf Acesso em: 25 set. 2021.

BUENO, F. Uso Irracional de Medicamentos: um agravo à Saúde Pública. 2017. 40f. Trabalho de Conclusão de Curso (Graduação em Farmácia) - Departamento de Ciências da Vida, Universidade Regional do Noroeste do Estado do Rio Grande do Sul - UNIJUÍ, Ijuí,RS. Disponível em: 
https://bibliodigital.unijui.edu.br:8443/xmlui/bitstream/handle/123456789/4499/Fla vio \%2oBueno.pdf? sequence $=\mathrm{I} \&$ is Allowed $=y$ Acesso em: I8 ago. 2021.

CARIAS, Claudia Mezleveckas et al. Medicamentos de dispensação excepcional: histórico e gastos do Ministério da Saúde do Brasil. Revista de Saúde Pública [online]. 20II, v. 45, n. 2 [Acessado 23 Agosto 202I] , pp. 233-240. Disponível em: $<$ https://doi.org

DELFINO, Marcus Ferreira. Consumo excessivo de medicamentos, um problema de saúde pública. Ret-sus, agosto-setembro 2012. Disponível em: http://scielo.com.br. Acesso em: 20 julho de 2021.

GANDOLFI, E.; ANDRADE, M. da G. G. Eventos toxicológicos relacionados a medicamentos no Estado de São Paulo. Revista de Saúde Pública, São Paulo, v.4o, n.6, p.1056-1064, 2006. Disponível em: https://www.scielo.br/scielo.php?script=sci_arttext\&pid=So034-89102006000700014 Acesso em: Io set. 2021.

GERMANO, L. C.; ALONSO, H. G. A. Estudo descritivo dos atendimentos hospitalares por eventos toxicológicos em um município do estado de São Paulo, 2012. Epidemiologia e Serviços de Saúde, Brasília, v.26, n.3, jul./set., 2017.Disponível em: $\quad$ https: $/ /$ www.scielo.br/scielo.php?script $=$ sci_arttext $\&$ pid $=$ S2237- $_{\text {- }}$ 96222017000300545 Acesso em: 1o set. 2021.

GONÇALVES, C. A. et al. Intoxicação Medicamentosa: relacionada ao uso indiscriminado de medicamentos. Revista Científica da Faculdade de Educação e Meio Ambiente, Ariquemes, RO, v.8, n.I, p.135-143, jan./jun., 2017. Disponível em: http://www.faema.edu.br/revistas/index.php/Revista-

FAEMA/article/view/449/442 Acesso em: I3 ago. 2021.

LUCHESSI, André Ducati. MARÇAL, Bruno Fonseca. ARAÚJO, Geraldo Franca de. ULIANA, Lívia Zini. ROCHA, Marilia Reis Gonçalves. PINTO. Terezinha de 
Jesus Andreoli. Monitoração de propaganda e publicidade de medicamentos: âmbito de São Paulo. Revista Brasileira de Ciências Farmacêuticas [online]. 2005, v. 4I, n. 3 [Acessado 2. Setembro 202I] , pp. 345-349. Disponível em: $<$ https://doi.org.

MACEDO, G. R. et al. O poder do marketing no consumo excessivo de medicamentos no Brasil. Transformar, p.II4-I28, 2016. Disponível em: http://www.fsj.edu.br/transformar/index.php/transformar/article/view/79/75 Acesso em: 05 set. 2021.

MARGONATO, Fabiana BURDINI, Thomson, Zuleika e PAOLIELLO, Mônica Maria Bastos. Determinantes nas intoxicações medicamentosas agudas na zona urbana de um município do Sul do Brasil. Cadernos de Saúde Pública [online]. 2008, v. 24, n. 2 [Acessado 2 Setembro 202I], pp. 333-34I. Disponível em: <https://doi.org

MINISTÉRIO DA SAÚDE.Talidomida- orientação para uso controlado 2013. Disponível em: <www.saude.gov.br. Acesso: Io julho 2021.

NASCIMENTO, Marilene Cabral do. Medicamentos: ameaça ou apoio à saúde? Rio de Janeiro: Vieira e Lent; 2003200 p. graf. Monografia em Português | LILACS | ID: 1 il- 348872

PAUlA, Claudia Costa da Silva. CAMPOS, Renata Bernardes Faria. SOUZA, Maria Celeste Reis Fernandes de. Uso irracional de medicamentos: uma perspectiva cultural. Brazilian Journal of Development, Curitiba, v.7, n.3, p. 2166o-21676 mar 2020 .

PORTA, Valentina. Farmacologia Clínica: Fundamentos da terapêutica racional. Revista Brasileira de Ciências Farmacêuticas [online]. 2006, v. 42, n. 4 [Acessado 23 Agosto 2021] , pp. 616-617. Disponível em: <https://doi.org 
RIBEIRO, A. T. et al. O Perfil das intoxicações medicamentosas no Brasil. Brazilian Journal Development, Curitiba, v.6, n.3, p.9632-9634, mar., 2020. Disponível em: https://www.brazilianjournals.com/index.php/BRJD/article/view/7259/6308 Acesso em: is set. 2021.

ROMANO-LIEBER, Nicolina Silvana. TEIXEIRA, Jorge Juarez Vieira. FARHAT, Fatima Cristiane Lopes Goularte. RIBEIRO, Eliane. CROZATTI, Márcia Terezinha Lonardoni. OLIVEIRA, Giane Sant'Ana Alves de. Revisão dos estudos de intervenção do farmacêutico no uso de medicamentos por pacientes idosos. Caderno de Saúde Pública, n. 6, v. 18, p. 1459-1507, 2002.

SALOMÃO, Rodinei Seba. Automedicação. Revista da Associação Médica Brasileira, v. 47, n. 4, Editorial, 200I. Disponível em http://scielo.com.br. Acesso em: I7 Mar.2021.

SANTOS, Jessica. Consumo excessivo de medicamentos, um problema de saúde pública. Ret-sus, agosto-setembro 2012. Disponível em: www.retsus.epsjv.fiocruz.br. Acesso em 02 de set. 2021.

TOURINHO, Francis Solange Vieira. Automedicação em crianças e adolescentes: Inquérito Populacional nos Municípios de Limeira e Piracicaba, Estado de São Paulo. Campinas, SP: 2008. Originalmente apresentada como dissertação de Doutorado, Universidade Estadual de Campinas, 2008.

VIEIRA, F. S.; ZUCCHI, P. Financiamento da assistência farmacêutica no sistema único de saúde. Saúde e Sociedade. São Paulo,v.22, n.I, p.73-84, 2013. Disponível em: https://www.scielo.br/scielo.php?script=sci_arttext\&pid=SoI04- I2902013000100008 Acesso em: 03 set. 2021. 